\title{
Synchronization of two different chaotic systems via nonlinear control
}

\author{
*11BABALOLA, MI; IYORZOR, BE \\ University of Benin Benin City, Edo State, Nigeria \\ Email Address: mib4real2003@yahoo.com Tel :+2348060797955
}

\begin{abstract}
This work reports the synchronization of a pair of four chaotic systems via nonlinear control technique. This method has been found to be easy to implement and effective especially on two different chaotic systems. We paired four chaotic systems out of which one is new and we have six possible pairs. Our numerical results show how effective the nonlinear control method is to chaotic systems. ( ) JASEM

http://dx.doi.org/10.4314/jasem.v21i1.10
\end{abstract}

Keyword: Synchronization, nonlinear control, chaos, attractors, controllers, secure communications

Introduction: Nonlinear dynamical systems with high sensitivity to initial conditions are termed chaotic. The chaotic behavior of these systems can be found in real life situation and also in devices (Singer et al., 1991). There are many features of chaotic systems which have been studied and they include chaos control, chaos stability, pattern formation, amplitude death, chaos synchronization etc. Ever since the introduction of the field of chaos synchronization by Pecora and Carroll (1990), the field has attracted the attention of many researchers (Ajayi et al., 2014; Laoye et al., 2008; Yang, 2012; Lu et al., 2013; Ho and Hung, 2002; Masoller, 2001; Park, 2006; Lu and $\mathrm{Lu}, 2003)$ due to its application in electronics, secure communications, modeling brain and cardiac rhythmic activity etc. Because of the importance of synchronization in theory and practical applications, several synchronization techniques have been studied which include projective, complete, generalized, anticipated and adaptive synchronization. Synchronization of identical chaotic systems is common in theory (Idowu and Vincent, 2013; Olusola et al., 2011), but in practical world, most systems cannot be assumed to be identical especially in laser arrays (Park, 2006). Although the synchronization of two different chaotic systems have been reported using different methods, we have employed the nonlinear control method on four chaotic systems in order to investigate the effectiveness of the method on chaotic systems.

System Description: In this Section, we considered pair of four chaotic systems in which one is taken as the drive system and the other one is taken as the response system. When two systems are paired from four systems then we have six possibility of pairing. The four chaotic systems are:

Lu, Chen and Zhang system (Lu et al., 2002)

$\left\{\begin{array}{c}\dot{x}=a(y-x) \\ \dot{y}=-x z+c y \\ \dot{z}=x y-b z\end{array}\right.$

System (1) has chaotic attractor when $a=36, b=3, c=20$

Liu system (Liu et al., 2004)

$\left\{\begin{array}{c}\dot{x}=a(y-x) \\ y=b x-k x z \\ \dot{z}=-c z+h x^{2}\end{array}\right.$

System (2) has chaotic attractor when $a=10, b=40, c=2.5, h=4, k=1$

Chen system (Chen and Ueta, 1999)

$\left\{\begin{array}{c}\dot{x}=a(y-x) \\ y=(c-a) x-x z+c y \\ \dot{z}=x y-b z\end{array}\right.$

System (3) has chaotic attractor when $a=35, b=3, c=28$ 
Qi et al system (Qi et al., 2008)

$\left\{\begin{array}{c}\dot{x}=a(y-x)+e y z \\ y=c x+d y-x z \\ \dot{z}=-b z+x y\end{array}\right.$

System (4) has chaotic attractor when $a=14, b=43, c=-7, d=16, e=4$

Synchronization between different chaotic systems: For chaos synchronization between (1) and (2), (1) is taken as the drive system while (2) is taken as the response system having a new form (5) with control parameters $\mathrm{u}_{1}$, $\mathrm{u}_{2}, \mathrm{u}_{3}$

$\left\{\begin{array}{c}x_{1}^{\prime}=a_{1}\left(y_{1}-x_{1}\right)+u_{1} \\ y_{1}^{\prime}=b_{1} x_{1}-k_{1} x_{1} z_{1}+u_{2} \\ z_{1}^{\prime}=-c_{1} z_{1}+h_{1} x_{1}{ }^{2}+u_{3}\end{array}\right.$

Our target is to determine the control parameter $\mathrm{u}_{\mathrm{i}}$ for the global synchronization of system (1) and (5).

We define

$\left\{\begin{array}{l}e_{1}=x_{1}-x \\ e_{2}=y_{1}-y \\ e_{3}=z_{1}-z\end{array}\right.$

$\left\{\begin{array}{l}e_{1}^{\prime}=a_{1} y_{1}-a_{1} x_{1}-a y+a x+u_{1} \\ e_{2}^{\prime}=b_{1} x_{1}-k_{1} x_{1} z_{1}+x z-c y+u_{2} \\ e_{3}^{\prime}=-c_{1} z_{1}+h x_{1}^{2}-x y+b z+u_{3}\end{array}\right.$

If we choose

$\left\{\begin{array}{c}u_{1}=a_{1} x+y_{1}-y-a_{1} y_{1}+a y-a x \\ u_{2}=-b_{1} y_{1}-b_{1} y-b_{1} x_{1}+k_{1} x_{1} z_{1}-x z+c y \\ u_{3}=c_{1} z_{1}-h x_{1}{ }^{2}+x y-b z\end{array}\right.$

then

$\left\{\begin{array}{c}e_{1}^{\prime}=-a_{1} e_{1}+e_{2} \\ e_{2}^{\prime}=-b_{1} e_{2} \\ e_{3}^{\prime}=-c_{1} e_{3}\end{array}\right.$

The error dynamics of system (9) can be re-expressed in matrix form as

$P=\left[\begin{array}{ccc}-a_{1} & 1 & 0 \\ 0 & -b_{1} & 0 \\ 0 & 0 & -c_{1}\end{array}\right]$

where $a_{1}=10, b_{1}=40, c_{1}=2.5$. The eigenvales of $P$ are $-40,-2.5,-10$ which satisfy the Hurwitz criterion for systems to be asymptotically stable i.e. all eigenvalues must have negative real part. Once this is achieved then it implies that system (5) synchronizes system (1). If we represent the synchronization of system (1) and system (2) with $A_{1}$ then synchronization of system (1) and system (3) is $A_{2},(1)$ and (4) is $A_{3}$, (2) and (3) is $A_{4}$, (2) and (4) is $A_{5}$, and finally (3) and (4) with $A_{6}$. For $A_{1}$ the control parameters are given by (8) and the error dynamics are given by (9)

The following are the list of control parameters and error dynamics for the various pair of systems $A_{2}, A_{3}, A_{4}$, $\mathrm{A}_{5}$, and $\mathrm{A}_{6}$ after following the same procedure as in $\mathrm{A}_{1}$

For $\mathrm{A}_{2}$, the control parameters are as follows:

$$
\left\{\begin{array}{c}
u_{1}=\left(a_{1}-a\right) x+y_{1}\left(1-a_{1}\right)-y(a-1) \\
u_{2}=\left(a_{1}-c_{1}\right) x_{1}-x z-\left(b_{1}+c_{1}\right) y_{1}-\left(b_{1}+c\right) y+x_{1} z_{1} \\
u_{3}=c_{1}\left(z-z_{1}-y_{1}\right)+b_{1} z_{1}+x y-b z
\end{array}\right.
$$


And the error dynamics are

$\left\{\begin{array}{c}e_{1}^{\prime}=-a_{1} e_{1}+e_{2} \\ e_{2}^{\prime}=-b_{1} e_{2} \\ e_{3}^{\prime}=-c_{1} e_{3}\end{array}\right.$

Where $a_{1}=35, b_{1}=3, c_{1}=28, a=36, b=3$, and $c=20$. The eigenvalues of (12) are all negative real part which satisfy the Hurwitz criterion for systems to be asymptotically stable.

For $\mathrm{A}_{3}$, the control parameters are as follows:

$$
\left\{\begin{array}{c}
u_{1}=\left(a_{1}-a\right) x+y_{1}\left(1-a_{1}\right)-y(a-1)-e_{o} y_{1} z_{1} \\
u_{2}=-c y_{1}-c_{1} x_{1}+d_{1} y_{1}-x z+x_{1} z_{1} \\
u_{3}=b_{1} z-x_{1} y_{1}+x y-b z
\end{array}\right.
$$

And the error dynamics are

$$
\left\{\begin{array}{c}
e_{1}^{\prime}=-a_{1} e_{1}+e_{2} \\
e_{2}^{\prime}=-c e_{2} \\
e_{3}^{\prime}=-b_{1} e_{3}
\end{array}\right.
$$

Where $a_{1}=14, b_{1}=43, c_{1}=-7, d_{1}=16, e_{0}=4, a=36, b=3$, and $c=20$. The eigenvalues of (14) are all negative real part which satisfy the Hurwitz criterion for systems to be asymptotically stable.

For $\mathrm{A}_{4}$, the control parameters are as follows:

$\left\{\begin{array}{c}u_{1}=\left(a_{1}-a\right) x+y_{1}\left(1-a_{1}\right)-y(a-1) \\ u_{2}=b_{1} y-\left(b_{1}+c_{1}\right) y_{1}+\left(a-c_{1}\right) x+x_{1} z_{1}+x b+k x z \\ u_{3}=z_{1}\left(b_{1}-c\right)-y_{1} x_{1}+h x^{2}\end{array}\right.$

And the error dynamics are

$\left\{\begin{array}{c}e_{1}^{\prime}=-a_{1} e_{1}+e_{2} \\ e_{2}^{\prime}=-b_{1} e_{2} \\ e_{3}^{\prime}=-c e_{3}\end{array}\right.$

Where $a_{1}=35, b_{1}=3, c_{1}=28, a=10, b=40, c=2.5, k=1$ and $h=4$. The eigenvalues of $(16)$ are all negative real part which satisfy the Hurwitz criterion for systems to be asymptotically stable.

For $\mathrm{A}_{5}$, the control parameters are as follows:

$\left\{\begin{array}{c}u_{1}=\left(a_{1}+a\right) x+y_{1}+y(a-1)-a_{1} y_{1}-y_{1} e_{o} \\ u_{2}=-\left(b_{1}+d_{1}\right) y_{1}+b_{1} y-c_{1} x_{1}+x_{1} z_{1}+x b-k x z \\ u_{3}=z_{1}\left(b_{1}-c\right)-y_{1} x_{1}+h x^{2}\end{array}\right.$

And the error dynamics are

$\left\{\begin{array}{c}e_{1}^{\prime}=-a_{1} e_{1}+e_{2} \\ e_{2}^{\prime}=-b_{1} e_{2} \\ e_{3}^{\prime}=-c e_{3}\end{array}\right.$

Where $a_{1}=14, b_{1}=43, c_{1}=-7, d_{1}=16, e_{0}=4, a=10, b=40, c=2.5, k=1$ and $h=4$. The eigenvalues of $(18)$ are all negative real part which satisfy the Hurwitz criterion for systems to be asymptotically stable.

For $\mathrm{A}_{6}$, the control parameters are as follows:

$\left\{\begin{array}{c}u_{1}=\left(a_{1}-a\right) x_{1}+y_{1}\left(1-a_{1}\right)+y(a-1)-e_{o} y_{1} z_{1} \\ u_{2}=(c-a) x-\left(c-d_{1}\right) y_{1}+2 c y-c_{1} x_{1}+x_{1} z_{1}-x z \\ u_{3}=z_{1}\left(b_{1}-b\right)-y_{1} x_{1}+x y\end{array}\right.$

And the error dynamics are 
$\left\{\begin{array}{c}e_{1}^{\prime}=-a_{1} e_{1}+e_{2} \\ e_{2}^{\prime}=-c e_{2} \\ e_{3}^{\prime}=-b e_{3}\end{array}\right.$

Where $a_{1}=14, b_{1}=43, c_{1}=-7, d_{1}=16, e_{0}=4, a=35, b=3$, and $c=28$. The eigenvalues of $(20)$ are all negative real part which satisfy the Hurwitz criterion for systems to be asymptotically stable.

Numerical simulations: To verify the effectiveness of controllers (8), (11), (13), (15), (17) and (19), numerical solutions are presented. In the numerical simulation, the fourth-order Runge-Kutta method is used to solve the systems with time step size 0.001. Different initial conditions are used for the various pairs of systems.

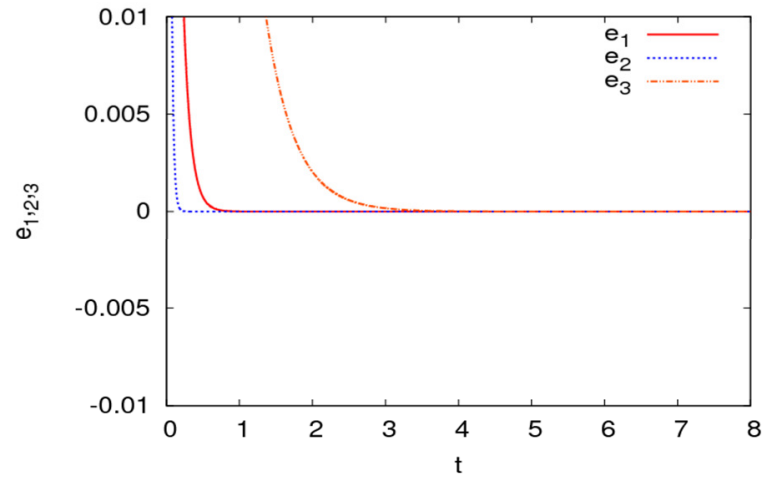

Fig. 1: Error dynamics of a pair of system $A_{1}$

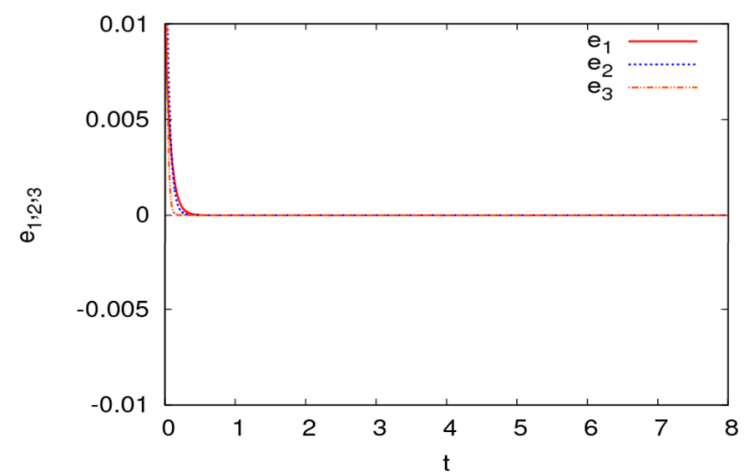

Fig. 3: Error dynamics of a pair of system $A_{3}$

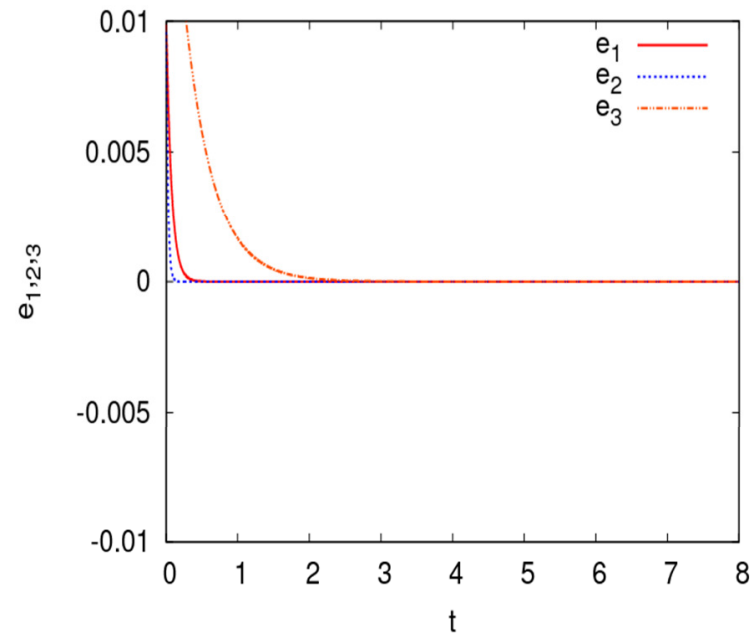

Fig. 5: Error dynamics of a pair of system $A_{5}$

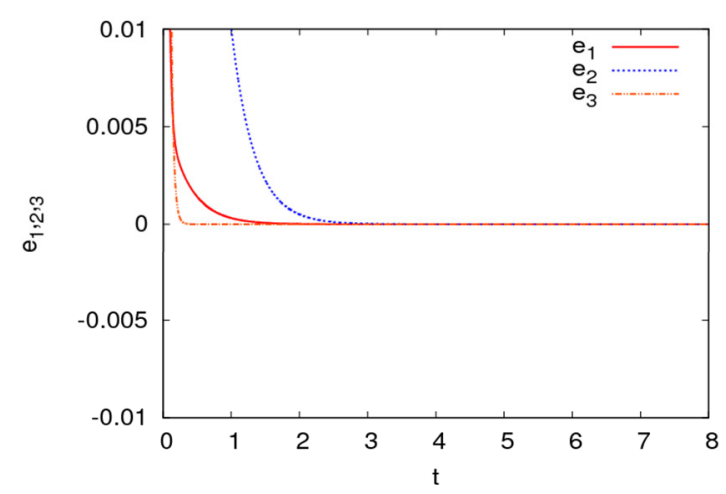

Fig. 2: Error dynamics of a pair of system $A_{2}$

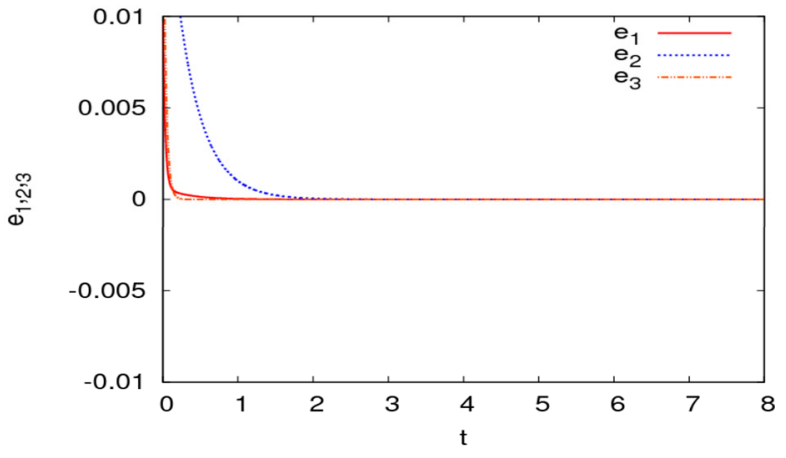

Fig. 4: Error dynamics of a pair of system $\mathrm{A}_{4}$

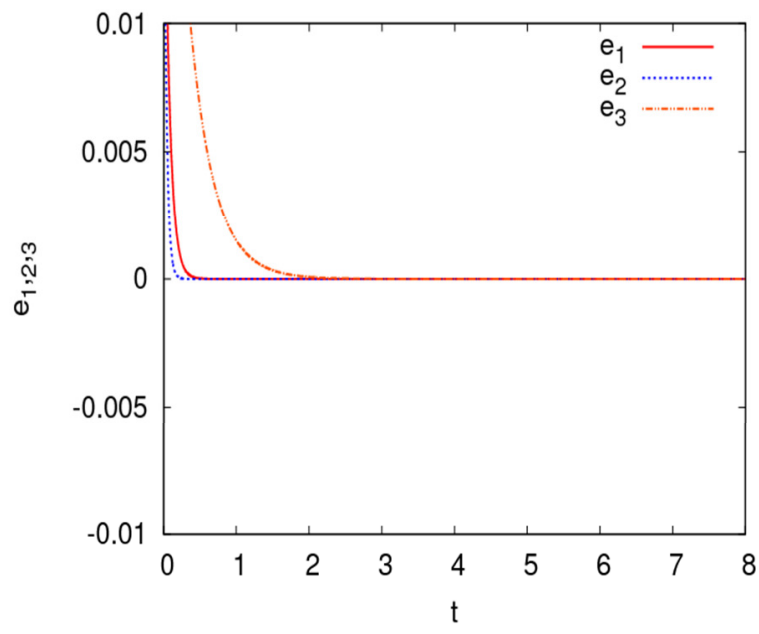

Fig. 6: Error dynamics of a pair of system $A_{6}$ 
Conclusion:We have been able to design control parameters via nonlinear control technique which is capable of synchronizing two different chaotic systems. This technique is effective and easy to implement as shown in our numerical results. The results of the six possible pair of chaotic systems are globally and asymptotically stable.

Acknowledgements:MIB is grateful to the Marie Curie Library of the Abdus Salam International Centre for Theoretical Physics (ICTP) for permission to use the eJDS facility for reference searching.

\section{REFERENCES}

Ajayi AA, Ojo SK, Vincent UE, Njah AN (2014). Multi-switching synchronizationof a driven hyperchaotic circuit using active backstepping. J. Nonl. Dynamics.2014:918586.

Chen G, Ueta T (1999). Yet another chaotic attractor. Int, J. Bifurcation and Chaos. 9:1465

Ho MC, Hung YC (2002). Synchronization of two different chaotic systems using generalized active network. Phys. Lett. A. 301:6424-8

Idowu BA, Vincent UE (2013). Synchronization and stabilization of chaotic dynamics in a quasi-1D Bose-Einstein condensate. Journal of Chaos. 2013:723581

Laoye JA, Vincent UE, Akigbogun OO (2008). Chaos control and reduced -order synchronization of the rigid body. Int. J. Nonlinear Sci. 6:106-113.

Liu C, Liu T, Liu L, Liu K (2004). A new cchaotic attractor.Chaos, Solitons\& Fractals. 22:10311038.
Lu J, Chen G, Zhang S (2002). The compound structure of a new chaotic attractor.Chaos, Solitons\& Fractals. 14:669-672.

Lu JH, Lu JA (2003). Conntrolling uncertain Lu system using linear feedback.Chaos, Solitons\& Fractals. 17:127-133.

Lu P, Wu Q, Yang Y (2013). Controlling transport and synchronization in non-identical inertia ratchets. J. Opt. Ther. And Application. 157:888899.

Masoller C (2001). Anticipation in the synchronization of chaotic semiconductor lasers with optical feedback. Phys.Rev. Lett. 86:2782-5

Olusola OI, Vincent UE, Njah AN, Ali E (2011).Controll and synchronization of chaos in biological systems via backsteping. Int. J. Nonlinear Sci. 11:121-8.

Park JH (2006). Chaos synchronization between two different chaotic dynamical systems.Chaos, Solitons\& Fractals. 27:549-554

Pecora LM, Carroll TL (1990) Synchronization of chaotic systems. Phys. Rev. Lett. 64:821-4

Qi G, Chen G, van Wyk MA, van Wyk BJ, Zhang Y (2008). A four-wing chaotic attractor generated from a new 3-D quadratic autonomous system. Chaos, Solitons\& Fractals. 38:705-721.

Singer J, Wang YZ, (1991). Bau HH. Controlling a chaotic system. Phys. Rev. Lett. 66:1123-5

Yang CC (2012). Robust synchronization and antisynchronization of identical $\phi 6$ oscillators via adaptive sliding mode control. J. Sound and Vibration. 331:501-9. 